This is the accepted manuscript of: A. Mingotti, L. Peretto, R. Tinarelli, A. Angioni, A. Monti and F. Ponci, "Calibration of Synchronized Measurement System: from the Instrument Transformer to the PMU," 2018 IEEE 9th International Workshop on Applied Measurements for Power Systems (AMPS), Bologna, 2018, pp. 1-5. doi: 10.1109/AMPS.2018.8494887

(C) 2018 IEEE. Personal use of this material is permitted. Permission from IEEE must be obtained for all other uses, in any current or future media, including reprinting/republishing this material for advertising or promotional purposes, creating new collective works, for resale or redistribution to servers or lists, or reuse of any copyrighted component of this work in other works 


\section{Calibration of Synchronized Measurement System: from the Instrument Transformer to the PMU}

\author{
A. Mingotti, L. Peretto, R. Tinarelli \\ Dept. of Electrical, Electronic and Information \\ Engineering \\ Alma Mater Studiorum - University of Bologna \\ Viale Risorgimento 2, Bologna, 40136, Italy
}

\author{
A. Angioni, A. Monti, F. Ponci \\ Institute for Automation of Complex Power Systems, \\ E.ON Energy Research Center, RWTH University \\ Mathieustrasse 10, 52074, Aachen, Germany
}

\begin{abstract}
Even if their deployment in electrical medium voltage networks was not as extensive as could be expected at the beginning, Phasor Measurement Units are key elements for Smart Grid operation. An impressive amount of papers discusses their implementation and usefulness in medium voltage network and almost as many deals with their metrological characterization. Although it is well known that instrument transformers are the main source of uncertainty in PMU-based synchronized measurements, this topic is not sufficiently tackled in the scientific literature. In this paper, the attention is focused on the calibration of a measurement system made by a PMU and a low-power instrument transformer commonly employed in the Italian distribution network.
\end{abstract}

Keywords-Phasor Measurement Unit, Smart Termination, Instrument Transformers, LPIT, Metrological characterization

\section{INTRODUCTION}

In order to accomplish the goals of modern distribution grids, such as automatic control of Distributed Energy Resources (DERs), upgrades in the current monitoring systems have to be undertaken. In particular, it is required to extend diffusely sensors over Medium Voltage (MV) and Low Voltage (LV) networks. This requires the installation of measurement devices with a trade-off among cost, considering the huge number of nodes and lines, and performance.

Recently, the Phasor Measurement Unit (PMU), is considered a key technology to enable advanced monitoring of distribution systems [1]. It measures the synchrophasor, which is of great support for accuracy and update rate of Distributed System State Estimation (DSSE) [2], frequency and Rate Of Change of Frequency (ROCOF) which can be exploited in Microgrid applications [3]. These measurements must be performed respecting the real time and accuracy requirements specified in the standard IEEE c37.118.1-2011 and its amendment IEEE c37.118.1a-2014 [4]. Moreover, they should also require low costs for installation in a rising number of distribution Medium and Low Voltage (MV and LV) buses. To this purpose, a LOw COst (LOCO) PMU may be integral part of a distribution automation system, therefore communicating via recognized standards for communication, such as IEC 61850 [5] and include cybersecurity measures. Under these specifications, the LOw COst (LOCO) PMU solution was developed [6] and used in this work.

$\mathrm{MV} / \mathrm{LV}$ sensors have to evolve and provide outputs directly connectable to acquisition systems. With this aim, [7] describes the so called "Smart Termination" (ST) installed in many secondary substations by Italian electrical utilities and described in the following chapters. Therefore, this paper aims at characterizing a novel measurement chain composed by: Low Power Instrument Transformer (LPIT), or ST, plus the LOCO PMU. In the literature there are some examples of metrological characterizations of the system PMU + instrument transformers such as [8] - [10]. No other work, to the knowledge of the authors, tackled a measurement chain containing this new kind of transformers, the LPITs [11-13]. Such measurement system will be then deployed, in the near future, in several Italian and European secondary substations.

The paper is structured as follows: Section II briefly describes the PMU and its features. In Section III the novel LPITs are described, while in Section IV the proposed setup for the characterization of the novel measurement chain is presented. Section V contains all the tests run for the metrological characterization of the setup, while Section VI lists all the tests results. Finally, in Section VII some comments and conclusions are presented.

\section{Phasor MEAsurement Unit}

\section{A. Hardware Description}

The LOCO PMU is made of three main components. The first is the data acquisition board, the MCC USB 201, which converts the analogue input (in a range $\pm 10 \mathrm{~V}$ ) to digital sampled values. Such board is set in order to acquire samples with a given sampling frequency, up to $12.5 \mathrm{kHz}$, when a specified trigger is received. In particular, by using the Pulse

Per Second (PPS) signal, received from the GPS unit, it is assured that the sampling window starts at regular intervals. The second important component is the measurement computation unit, the Raspberry Pi 3. It receives the samples via USB interface from the MCC USB 201 and process them via $\mathrm{C}++$ algorithms which extract synchrophasors, frequency and ROCOF. By exploiting the open-source libiec61850 libraries, the measurements are encapsulated in Sampled Value (SV) protocol and in UDP-IP packets, as required in the standard IEC 61850-90-5 [5] for PMU measurement exchange in Wide Area Networks (WAN). The messages are exchanged between the PMUs and the Phasor Data Concentrator (PDC) via an encrypted tunnel, via the open-source openVPN software. The third component is the GPS module, providing the PPS to the data acquisition board and the digital time information via Universal Asynchronous Receiver/Transmitter (UART) interface to the Raspberry PI. Considering other secondary components, the total hardware cost of the device is approximately 250 euros. This is why is considered low cost, compared to typical PMUs which cost more than thousand euro.

\section{B. The Algorithm}

The algorithm that runs regularly in the Raspberry Pi, permits to extract the measurements on a specified window, which is normally set to 1 second. When the PPS signal is received, the samples are acquired for an observation window shorter than 1 second. This is done in order to leave some time for the measurement computation and restart the acquisition before the next PPS is received. The samples are processed by a low pass filter, in this case a $3^{\text {rd }}$ order Butterworth filter with cut frequency equal to $70 \mathrm{~Hz}$, in order to remove high 
TABLE I. MECHANICAL AND ELECTRICAL SPECIFICATION OF THE COMBINED CURRENT AND VOLTAGE SENSOR

\begin{tabular}{|c|c|c|c|}
\hline Rated frequency & $50-60 \mathrm{~Hz}$ & $\begin{array}{c}\text { Rated primary } \\
\text { current }\end{array}$ & $300 \mathrm{~A}$ \\
\hline $\begin{array}{c}\text { Rated Primary } \\
\text { Voltage }\end{array}$ & $20 / \sqrt{3} \mathrm{kV}$ & Weight & $1.5 \mathrm{~kg}$ \\
\hline $\begin{array}{c}\text { Nominal Voltage } \\
\text { ratio in/out }\end{array}$ & $10000: 1$ & Accuracy class & 0.5 \\
\hline \multicolumn{2}{|c|}{ Nominal current ratio } & $1000 \mathrm{~A} / 31 \mathrm{mV}$ \\
\hline
\end{tabular}



Fig. 1 Schematic representation of the proposed setup

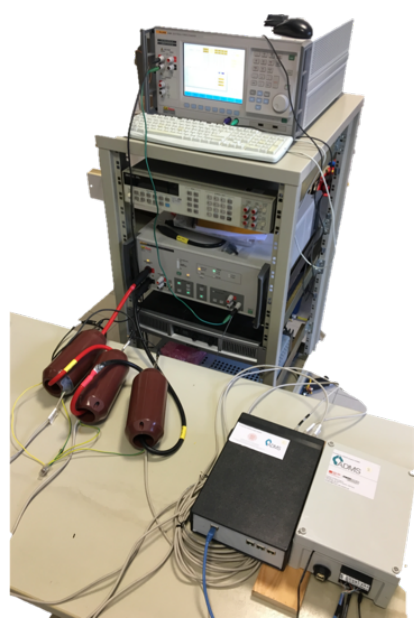

Fig. 2 Picture of the proposed setup during the measurements in the laboratory

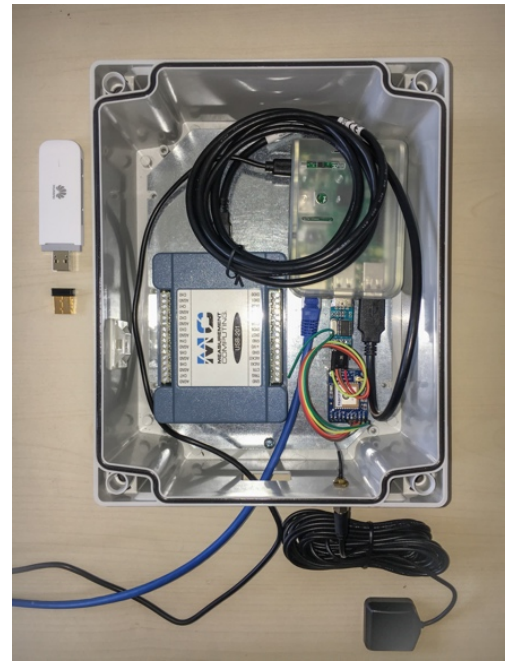

Fig. 3 Picture of the PMU highlighting the 3 main components

harmonics and noise content. The filtered samples are processed with a zero-crossing algorithm to extract the signal frequency. The ROCOF is obtained as difference between frequency measured at first and last cycle of the observation window. If the measured frequency is distant from the nominal $\left(f_{n} \pm 0.2 \mathrm{~Hz}\right)$ the Discrete Fourier Transform is calculated based on a reduced set of samples (only the first 6 cycles are selected). The DFT is applied on the original signal samples (not the ones filtered for the frequency calculation) multiplied by the flat top window. The phase angle is compensated considering the actual measured frequency in order to time tag the synchrophasor at the occurrence of the UTC second.

The described hardware and software were tested for compliance of steady state accuracy and requirements. As shown in [6], the Total Vector Error (TVE) in steady state conditions shows to be always below $0.3 \%$. Similar results were obtained for frequency and ROCOF errors.

\section{LOW POWER INSTRUMENT TRANSFORMER}

A new kind of Instrument Transformers (ITs) is replacing the legacy inductive ones: the LPITs. Therefore, new Standards were required to fully describe these new components, the low-power current and voltage transformers described in [11] and [12], respectively. LPITs features include lower weight and dimensions with respect to the inductive ones. Moreover, the novelty they introduce is the low-power output, ready for being connected, in most of the cases, to generic acquisition systems. Such characteristics make LPITs suitable for being spread and installed in many secondary substations, to acquire and have a better knowledge of the MV power network.

Authors' experience on LPITs have been consolidated with [14-15] and, with this work, a further step towards Smart Grids' implementation is done. The LPIT used in the following setup is a combined voltage and current sensor (CVS), suitable for MV indoor applications, which electrical and mechanical characteristics are summarized in Table I. The voltage is provided to the sensor through the high voltage electrode (top of the sensor) and the ground potential electrode (wire electrode). Concerning the current, the sensor is a trough-hole type; hence, the power cable is to be inserted into it. The output of the sensor is transmitted through RJ45 connector after the scaling procedure via the voltage and current transformers embedded in the sensor.

\section{MEASUREMENT SETUP}

A schematic representation of the proposed measurement setup is shown in Fig. 1. Fig. 2 instead, presents a picture of the measurement setup when tests have been performed. The setup consists of a 3-phase system with:

- Calibrator Fluke 6105A (maximum outputs: $1000 \mathrm{~V}$, $120 \mathrm{~A})$ under metrological confirmation, it provides $50 \mathrm{~Hz}$ sinusoidal voltage and current to feed the sensors.

- LPIT (Current and Voltage Sensor, CVS). Its characteristics are listed in Table I. The actual in/out ratios of the LPIT used to run tests are 8720 and 34598.5 for the voltage and the current, respectively.

- LOCO PMU: this low-cost version of the instrument allows to acquire phasors from a 3-phase system. It requires a time-reference signal to attach a time-stamp to the measurements. The inputs accepted are contained in the $\pm 10 \mathrm{~V}$ range. It is shown in Fig. 3 .

- Current and Voltage Adapter (CVA): this device, prototype developed in the lab, was necessary to adapt the output of the CVS, very low in particular for the current, to the input of the PMU. Its in/out ratios for both current and voltage correction are 240 and 4, 
TABLE II. AMPLITUDE MEASUREMENTS RESULTS: FROM CALIBRATOR TO PMU

\begin{tabular}{|c|c|c|c|}
\hline \#test & $\begin{array}{c}\text { Applied RMS } \\
\text { Voltage [V] }\end{array}$ & $\boldsymbol{V}_{\boldsymbol{M}}[\mathbf{V}]$ & $\boldsymbol{\sigma}_{\boldsymbol{V}_{\boldsymbol{M}}[\mathbf{V}]}$ \\
\hline $\mathbf{1}$ & 200 & 198.97 & 0.04 \\
\hline $\mathbf{2}$ & 600 & 600.62 & 0.04 \\
\hline $\mathbf{3}$ & 800 & 799.44 & 0.05 \\
\hline $\mathbf{4}$ & 1000 & 999.42 & 0.05 \\
\hline
\end{tabular}

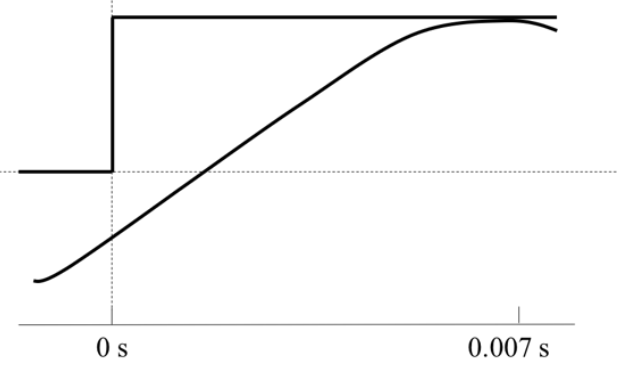

Fig. 4 Snapshot of the two waveforms acquired by the Oscilloscope for the phase measurement

respectively. The CVA has been characterized using the Fluke calibrator in terms of both, amplitude and phase. The results are not presented for the sake of brevity, however the phase angle deviations obtained are negligible.

- Oscilloscope Tektronix MSO5 series (see Fig. 1) Used to evaluate the phase delay between the pulse per second and the sine wave maxima. It features an accuracy of $2.5 \cdot 10^{-6}$ for time measurements. The mentioned test will be described in the following section.

In a nutshell, the calibrator fluke provides the input voltage and current signals to the sensors. Their output is provided to the CVA, which, in the field, feed the signal to the RGDM (Rilevatore di Guasto Direzionale e Misura) and LOCO PMU. The PMU publishes its results via IEC 61850, which are collected by a cloud phasor data concentrator. Finally, the PMU data are downloaded regularly, to compare them with the input data produced by the calibrator. The oscilloscope is used as additional reference for the knowledge of the phase angle of the waveforms.

\section{TESTS DESCRIPTION}

\section{A. Introduction}

In this Section, all the tests performed on the measurement chain (Fig. 1) are described. The intent is to evaluate the accuracy of magnitude, phase angle, frequency and ROCOF quantities measured by the system sensor + PMU. As already described, the system is to be applied in distribution systems, therefore the tests on frequency and ROCOF are limited in range and number with respect of the indications of the IEEE Std. C37.118.1-2011 and C37.118.1a-2014. Furthermore, in distribution grids, current and voltage phasors are elaborated to obtain power flow and injection measurements, therefore it is included in the tests the accuracy of the phase angle

This research has been partially funded by the European projects Horizon H2020 “ADMS: Smart Grid Active Distribution Management System to accommodate Renewable Energy Sources and Low Carbon Emissions" and SOGNO: Service Oriented Grid for the Network Of the future. difference between voltage and current. Phase angle measurements have to be repeated several times during the lab testing sessions. Such procedures require having a fixed phase angle to ensure the repeatability of the test over time. This however clashes with the rotation of the phase angle when the frequency generated by the calibrator is not exactly $50 \mathrm{~Hz}$. Such effects are negligible when the test are run over short period of times, e.g. few seconds, but become preeminent when the test run for hours. Therefore this test are conducting adjusting the time reference that is sent to the PMU to trigger the acquisition to be a multiple of the signal frequency and not necessary a multiple of $50 \mathrm{~Hz}$. For instance, if the generated signal matches nominal frequency is $50 \mathrm{~Hz}$ the time pulse is refreshed every 1 second (this will match the PPS signal), if instead the generated signal is $49.995 \mathrm{~Hz}$, the time pulse is refreshed every 0.99999 seconds. This method allows the PMU to generate a phasor that rotates in the same domain of the current system frequency.

It is worth noticing that the Total Vector Error (TVE), as defined in the IEEE Std. C37.118.1-2011 is also not commonly applied in distribution network monitoring applications. Therefore, the errors are always split in terms of magnitude and phase angle error and quantified statistically as average + standard deviation

\section{B. Tests}

First of all, the amplitude of the quantities acquired by the PMU was verified. To do so, different values of voltage were set by the calibrator $(200,600,800,1000 \mathrm{~V})$, which feature, for the most interesting range $(1000 \mathrm{~V})$, an accuracy of $60 \mathrm{ppm}$ $+10 \mathrm{mV}$. For each input value of the voltage and current 1000 values are acquired from the PMU.

The second and third tests regard the phase of the acquired quantities. The former consisted in the absolute phase measurement of a voltage, with respect to the cosine waveform with positive peak aligned with the PPS signal (see Fig. 4). The phase was measured 1000 times with both the PMU and the Oscilloscope, and the results compared. This test aimed at verifying the quality of the time measurement of the PMU, using the Oscilloscope as a reference. The latter test dealt with the evaluation of the phase difference between the voltage and current. Three different phases were set for the current by the calibrator $(0, \pi / 4, \pi / 2 \mathrm{rad})$. As mentioned in the introduction, phase measurement test requires fixed values of phase over time. However, to avoid phase drifts, the aforementioned procedure has been applied, providing to the PMU a stable phase reference.

Finally, given that PMU allows frequency measurements, tests on frequency and ROCOF measurement have been performed. For three days, 1000 samples of both frequency and ROCOF have been acquired by the PMU. For all the aforementioned tests, the calibrator maintains the input signal for a fixed time window of $1 \mathrm{~h}$. In this way, it is possible to be confident that, even if the input signal of the calibrator is not timestamped, both calibrator and PMU are referring to the same signal.

\section{RESULTS}

In Tables II to $\mathrm{V}$ all measurement results are presented. In Table II four voltage RMS magnitudes measured by the PMU are compared with the calibrator input. The Table list the mean value $V_{M}$ of 1000 measurements together with the standard deviation of the mean $\sigma_{V_{M}}$. Assuming the magnitude error as

unique contributor to the for the system sensor + PMU system, we would have a TVE ranging from $0.5 \%$ (for $200 \mathrm{~V}$ 
TABLE III. PHASE MEASUREMENT RESULTS: ABSOLUTE PHASE COMPARISON BETWEEN OSCILLOSCOPE AND PMU

\begin{tabular}{|c|c|c|c|c|c|}
\hline \#day & $\boldsymbol{\varphi}_{\boldsymbol{O} \text { S }}[\mathbf{r a d}]$ & $\begin{array}{c}\boldsymbol{\sigma}_{\boldsymbol{\varphi}_{\boldsymbol{O}}} \\
{[\mathbf{r a d}]}\end{array}$ & $\begin{array}{c}\boldsymbol{\varphi}_{\boldsymbol{P M U}} \\
{[\mathbf{r a d}]}\end{array}$ & $\begin{array}{c}\boldsymbol{\sigma}_{\boldsymbol{P M U}} \\
{[\mathbf{r a d}]}\end{array}$ & $\Delta \boldsymbol{\varphi}[\mathbf{r a d}]$ \\
\hline $\mathbf{1}$ & 0.7627787 & $3 \cdot 10^{-7}$ & 0.7504 & $2 \cdot 10^{-4}$ & -0.0124 \\
\hline $\mathbf{2}$ & 2.1472786 & $2 \cdot 10^{-7}$ & 2.1366 & $2 \cdot 10^{-4}$ & -0.0107 \\
\hline $\mathbf{3}$ & 3.8798041 & $1 \cdot 10^{-7}$ & 3.8622 & $1 \cdot 10^{-4}$ & -0.0176 \\
\hline
\end{tabular}

TABLE IV. PHASE MEASUREMENT RESULTS: PHASE DIRRENCE BETWEEN THE VOLTAGE AND CURRENT PHASORS

\begin{tabular}{|c|c|c|c|}
\hline \#test & $\begin{array}{c}\text { Applied } \\
\text { Shift [rad] }\end{array}$ & $\begin{array}{c}\boldsymbol{\varphi}_{\boldsymbol{M}} \\
{[\mathbf{r a d}]}\end{array}$ & $\begin{array}{c}\boldsymbol{\sigma}_{\boldsymbol{\varphi}_{\boldsymbol{M}}} \\
{[\mathbf{r a d}]}\end{array}$ \\
\hline $\mathbf{1}$ & $1.571(\pi / 2)$ & 1.589 & 0.002 \\
\hline $\mathbf{2}$ & $0.785(\pi / 4)$ & 0.749 & 0.006 \\
\hline $\mathbf{3}$ & 0.0000 & 0.023 & 0.0008 \\
\hline
\end{tabular}

TABLE V. FREQUENCY MEASUREMENT RESULTS: FREQUENCY AND ROCOF EVALUATION

\begin{tabular}{|c|c|c|c|c|c|}
\hline \#day & $\begin{array}{c}\text { Applied } \\
\text { Frequency } \\
{[\mathbf{H z}]}\end{array}$ & $\boldsymbol{f}_{\boldsymbol{M}}[\mathrm{Hz}]$ & $\begin{array}{c}\boldsymbol{\sigma}_{\boldsymbol{f}_{\boldsymbol{M}}} \\
{[\mathbf{H z}]}\end{array}$ & $\begin{array}{c}\text { ROCOF } \\
{[\mathbf{H Z} / \mathbf{s}]}\end{array}$ & $\begin{array}{c}\boldsymbol{\sigma}_{\boldsymbol{R} O \boldsymbol{C}} \\
{[\mathbf{H z} / \mathbf{s}]}\end{array}$ \\
\hline $\mathbf{1}$ & 50 & 50.001209 & $3 \cdot 10^{-6}$ & 0.0034 & $4 \cdot 10^{-4}$ \\
\hline $\mathbf{2}$ & 50 & 50.001200 & $6 \cdot 10^{-5}$ & $\begin{array}{c}0.00020 \\
0\end{array}$ & $4 \cdot 10^{-6}$ \\
\hline $\mathbf{3}$ & 50 & 50.001225 & $7 \cdot 10^{-5}$ & 0.058 & $2 \cdot 10^{-3}$ \\
\hline
\end{tabular}

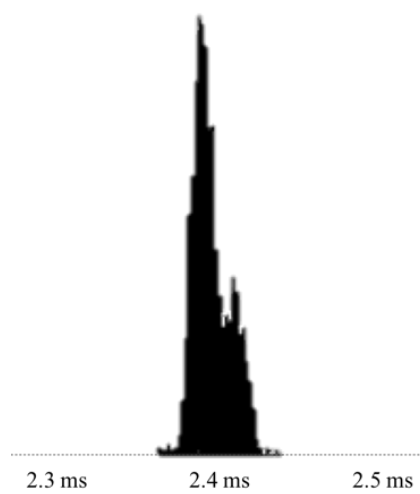

Fig. 5 Pdf of the voltage phase acquired with the oscilloscope

input) to $0.06 \%$ (for $1000 \mathrm{~V}$ ), thus being in line with the requisite for accuracy of synchrophasors (even in presence of the sensor uncertainty).

Table III list the 3 days measurements values of: the absolute phase angle measured with the oscilloscope $\varphi_{O s}$ and the one measured by the PMU $\varphi_{P M U}$. Both values are reported with their mean standard deviation $\sigma_{\varphi_{O S}}$ and $\sigma_{P M U}$, respectively. Moreover, in the Table the difference between the two mean values $\Delta \varphi$ is reported to improve the results comprehension. The corresponding TVE, assuming the phase angle error as only contributor to the TVE, for the sensor + PMU system, would be between $0.12 \%$ and $0.1 \%$.

The results of the phase difference between the voltage and current phasors measurements are listed in Table IV. The figures are the results of 1000 acquisitions, as for all the tests performed. $\varphi_{M}$ is the phase difference acquired by the PMU and $\sigma_{\varphi_{M}}$ its mean standard deviation. The results confirm what has been already stated in the previous phase measurement test. In Fig. 5 the probability density function of a phase measurement has been reported to confirm its normality distribution.
Finally, in Table $\mathrm{V}$ the frequency measurements are reported: aside from the applied frequency, the Table reports 3 days of measurement for the frequency $f_{M}$ acquired by the PMU and its ROCOF. All results are provided with their mean standard uncertainty $\sigma_{f_{M}}$ and $\sigma_{f_{M}}$, respectively. The frequency error is in the range of $1 \mathrm{mHz}$, in line with the conventional limits specified for frequency measurements for synchrophasors.

\section{CONCLUSIONS}

The system proposed for monitoring distribution grids shows accurate results, in line with common sensor and synchrophasor standards. In fact, the system sensor + PMU would satisfy both an accuracy class 0.5 for sensor standard and the 1\% TVE for PMU standard. However, it is required to advance the definition of the standard testing procedure and requirements for combined chain sensor plus devices. The test procedure should also take into account the peculiar features of distribution grids and not just extend the same rules applied to transmission. In this sense, this paper proposes a first set of tests that could become a benchmark for the LPIT + PMU chain characterization. In particular the phase angle is tested as absolute and relative information (phase angle of voltage minus current) by fixing the time reference to the signal frequency and not the nominal frequency.

\section{REFERENCES}

[1] Monti, A. Muscas, C. Ponci, F. "Phasor Measurement Units and Wide Area Monitoring Systems", Book, 2016, Elsevier Science \& Technology Books.

[2] C. Muscas, S. Sulis, A. Angioni, F. Ponci and A. Monti, "Impact of Different Uncertainty Sources on a Three-Phase State Estimator for

[3] Distribution Networks," in IEEE Transactions on Instrumentation and Measurement, vol. 63, no. 9, pp. 2200-2209, Sept. 2014.

[4] E. De Din, G. Lipari, A. Angioni, F. Ponci and A. Monti, "Effect of the reporting rate of synchrophasor measurements for distributed secondary control of AC microgrid," 2017 IEEE International Workshop on Measurement and Networking (M\&N), Naples, 2017, pp. 1-6.

[5] IEEE Standard for Synchrophasor Measurements for Power Systems, in IEEE Std C37.118.1-2011 (Revision of IEEE Std C37.118-2005), vol., no., pp.1-61, Dec. 282011.

[6] IEC 61850: 2018, "Communication networks and systems for power utility automation", International Standardization Organization, Geneva, Switzerland, 2018.

[7] A. Angioni, G. Lipari, M. Pau, F. Ponci and A. Monti, "A Low Cost PMU to Monitor Distribution Grids," 2017 IEEE International Workshop on Applied Measurements for Power Systems (AMPS), Liverpool, 2017, pp. 1-6.

[8] I. Gentilini, R. Calone, F. Giammanco, G. Bolcato, J. Weichold, M. Stalder, "The smart termination: An innovative component to enable Smart Grids development",22 $2^{\text {nd }}$ International conference and Exhibition on Electricity Distribution (CIRED), Stockholm, June 2013.

[9] M. Asprou, E. Kyriakides, M. Albu, "The effect of PMU measurement chain quality on line parameter calculation", IEEE International Instrumentation and Measurement Technology Conference, Turin, May 2017.

[10] R. S. Singh, S. Cobben, M. Gibescu, "Assessment of errors in the measurement chain of distribution grids feasibility study of a PMU application", First International Colloquium on Smart Grid Metrology, Split, Apr. 2018.

[11] "Uncertainty propagation in PMU-based trasmission line monitoring", IET Generation, Transmission \& Distribution, vol. 12, no. 3, pp. 745755 , Feb. 2018

[12] IEC 61869-10:2011, "Instrument transformers - Part 10: Specific requirements for low-power passive current transformers", International Standardization Organization, Geneva, Switzerland, 2017 . 
[13] IEC 61869-11:2011, "Instrument transformers - Part 11: Additional requirements for low-power passive voltage transformers", International Standardization Organization, Geneva, Switzerland, 2016

[14] A. Mingotti, L. Peretto, R. Tinarelli, K. Yigit, "Simplified Approach to Evaluate the Combined Uncertainty in Measurement Instruments for Power Systems", IEEE Transaction on Instrumentation and Measurement, pp. 2258-2265, vol. 66, Issue n.9, 2017.
[15] A. Mingotti, L. Peretto, R. Tinarelli, A. Ghaderi, "Uncertainty sources analysis of a calibration system for the accuracy vs. temperature verification of voltage transformers", paper accepted to IMEKO 2018, Belfast

[16] A. Mingotti, L. Peretto, R. Tinarelli, "Low Power Voltage Transformer Accuracy Class Effects on the Residual Voltage Measurement", IEEE proc. of I2MTC, pp. 1308-1313, Houstont, 2018. 